\title{
SIMULADOR DE PROCESSOS INDUSTRIAIS PARA TESTE DE ALGORITMOS DE CONTROLE
}

DOI: 10.37702/2175-957X.COBENGE.2021.3606

Francieli Cristina - francieli.cs17@gmail.com

Instituto Federal de Santa Catarina

Rua Sergio Cella 91

89810-812 - Chapecó - SC

Rômulo Lira Milhomem - romulo.milhomem@ifsc.edu.br Instituto Federal de Santa Catarina

89813-000 3450D

89813-000 - Chapecó - SC

Leonardo José Fiori - leonardo.jf@aluno.ifsc.edu.br

Instituto Federal de Santa Catarina

R. Campinas 84

89805-697 - CHAPECO - SC

João Pedro Brunoni - joao.pb08@aluno.ifsc.edu.br

Instituto Federal de Santa Catarina

Sete de Setembro 153

89802-220 - Chapecó - SC

Resumo: Boa parte do tempo gasto no desenvolvimento de sistemas de automação é no período de implementação, pois para que esse sistema esteja em pleno funcionamento é necessário a realização de diversos testes. Uma ferramenta muito útil e que possibilitaria reduzir esse tempo é o simulador, utilizado nas mais diversas áreas, ele possibilita testar e validar um processo sem a necessidade de se estar junto ao sistema real. Sob esse contexto, o artigo apresenta um simulador desenvolvido em um Controlador Lógico Programável capaz de permitir ao usuário prever o comportamento de um processo frente ao algoritmo de controle inserido, o que possibilitaria a diminuição no tempo de implementação do controle nas indústrias. O simulador recebe sinais de entrada de um segundo Controlador Lógico Programável e envia uma resposta dinâmica para o mesmo. A principal característica desse simulador é permitir ao usuário alterar parâmetros específicos de acordo com o sistema que se deseja simular, para isto ele conta com uma 


\section{(C. COBENGE e IV Simpósio Internacional

interface onde o usuário poderá não só alterar esses parâmetros, mas observar o comportamento gerado.

Palavras-chave: Aplicações Industriais, Automação e Controle de Processos, Simuladores de processos, Controladores Lógicos Programáveis. 


\section{SIMULADOR DE PROCESSOS INDUSTRIAIS PARA TESTE DE ALGORITMOS DE CONTROLE}

\section{INTRODUÇÃO}

Os softwares simuladores são capazes de prever o comportamento de um determinado sistema utilizando um computador como ferramenta, o que permite a elaboração de testes de funcionamento sem a necessidade de se utilizar o sistema real. Muitas vezes a realização de testes nos processos industriais podem acarretar em perdas de produção ou risco a segurança, assim a utilização deste tipo de ferramenta, é importante para verificar o comportamento do sistema, até mesmo, inibir esses fatores de perda ou risco na fase da implementação dos sistemas de automação.

No âmbito da indústria, principalmente na linha de produção, a simulação é aplicada com a finalidade de prever o impacto que uma determinada mudança na produção acarreta no sistema como um todo, ou seja, permite ao supervisor ter uma visão sistêmica que uma alteração local poderá gerar no contexto global do sistema (SANTORO e MORAES, 2000). Essa capacidade de prever um comportamento sem a necessidade de perturbá-lo, no caso de um sistema já implementado, é uma das vantagens de se utilizar a simulação na automação de processos, já que a maioria do que se desenvolve nessa área é aplicado a plantas industriais que já estão em funcionamento e que muitas vezes permitem pouca ou nenhuma parada para realização de testes.

Simulações de processos podem ser realizadas de pelo menos duas formas, offline ou online. No primeiro caso, pode-se utilizar um software de simulação matemática e simular o funcionamento dinâmico do processo a partir de sinais de entrada conhecidos, aplicados a modelos representados por equações diferenciais ou funções de transferência (OGATA, 2011). Caso o sistema seja de eventos discretos, pode-se utilizar uma abordagem por Autômatos ou Redes de Petri (CARDOSO e VALETTE, 1997). Por exemplo, para o funcionamento de uma máquina de fresagem em um processo de manufatura que sofre alterações bruscas, tais como início e fim da fresagem, cada mudança no estado da máquina é um evento discreto e, portanto, pode ser modelado como tal (CARDOSO e VALETTE, 1997).

$\mathrm{Na}$ simulação online utiliza-se um software (ou hardware) onde a dinâmica a ser simulada está implementada. Neste caso, observa-se a resposta do sistema simulado a sinais de entrada aplicados em tempo real. Assim, o objetivo desse estudo é desenvolver um software capaz de simular a dinâmica de um determinado processo industrial, possibilitando o teste de algoritmos de controle de forma online. O processo a ser simulado é um sistema industrial de nível de líquidos em tanques, que foi escolhido por ser um dos mais importantes na indústria (TRIGUEIRO e LIRA, 2014). Na sequência, será abordado a metodologia utilizada para simulação do sistema proposto, as características do sistema simulado e a obtenção do modelo matemático e sua validação.

\section{SISTEMAS E SIMULAÇÃO}

A Figura 1 ilustra uma representação esquemática de um sistema de nível em um reservatório de água. Sistemas que dispõem dessa configuração são bastante utilizados em estações de tratamento de água e de efluentes, porém existem outros segmentos onde esse tipo de processo também é aplicado, tais como indústria petroquímica, nuclear e de celulose (TRIGUEIRO e LIRA, 2014). 
Figura 1 - Diagrama do Sistema de Nível.

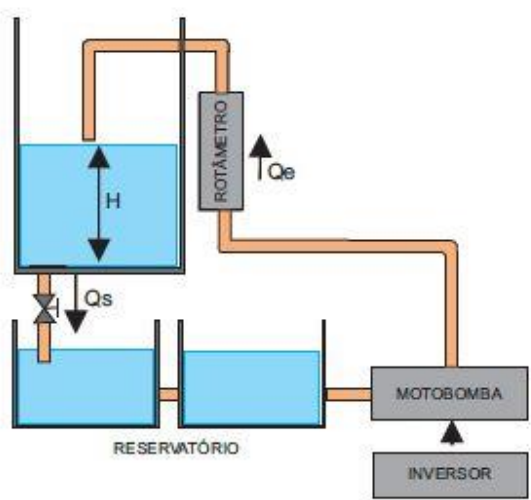

Fonte: Autoria Própria.

As características do sistema simulado bem como as do software desenvolvido são apresentadas a seguir.

\subsection{Caracterização do sistema simulado}

O sistema simulado utiliza uma bomba hidráulica centrífuga como atuador. Optou-se por essa configuração pois é o que geralmente é utilizado nos processos industriais, e está disponível no sistema da Figura 2, que será abordado adiante. Outra consideração sobre o sistema está em relação à área do tanque considerada constante, assim como a definição da água como líquido utilizado. O hardware utilizado para desenvolvimento do simulador é um Controlador Lógico Programável (CLP), escolhido por conta de sua aplicabilidade em processos industriais. Além disso, busca-se testar o CLP que será futuramente utilizado na aplicação de um sistema de controle industrial, o que possibilita o teste do conjunto hardware e software para sua implementação.

Assim, para testes do algoritmo de controle, o CLP simulador é conectado a um CLP secundário cuja aplicação a ser testada está implementada. A simulação é configurável para outros tipos de sistemas de nível, tamanhos de tanques e bombas. Para tanto, é necessário que alguns parâmetros sejam definidos pelo usuário, o sistema conta com uma Interface Homem Máquina (IHM) que possibilita essa interação. Para testar os algoritmos produzidos foi necessário utilizar um sistema de nível de uma bancada didática disponível no Senai de Chapecó - SC (Figura 2), a fim de comprovar os resultados obtidos e avaliálos em um sistema real. A Tabela 1 descreve os componentes presentes na bancada de acordo com a numeração dos itens destacados na Figura 2. A frequência do inversor determina a velocidade do motor e é alternada a partir de um sinal de comando em corrente, na faixa de 4 a $20 m A$.

Tabela 1 - Componentes da Bancada.

\begin{tabular}{c|c}
\hline Número & Componente Bancada \\
\hline 01 & Inversor Sinamics V20 (SIEMENS 2015) \\
\hline 02 & CLP S71200 Siemens \\
\hline 03 & Sensor de nível ultrassônico \\
\hline 04 & Rotâmetro \\
\hline 05 & Tanque principal \\
\hline 06 & Tanques secundários \\
\hline 07 & Motobomba centrífuga BC91-S - Schneider (ELETRIC e SIEMENS, s.d.) \\
\hline 08 & Painel Elétrico \\
\hline
\end{tabular}

Fonte: Autoria Própria. 
Figura 2 - Bancada didática de controle de nível utilizada nos experimentos e painel de comando, respectivamente.

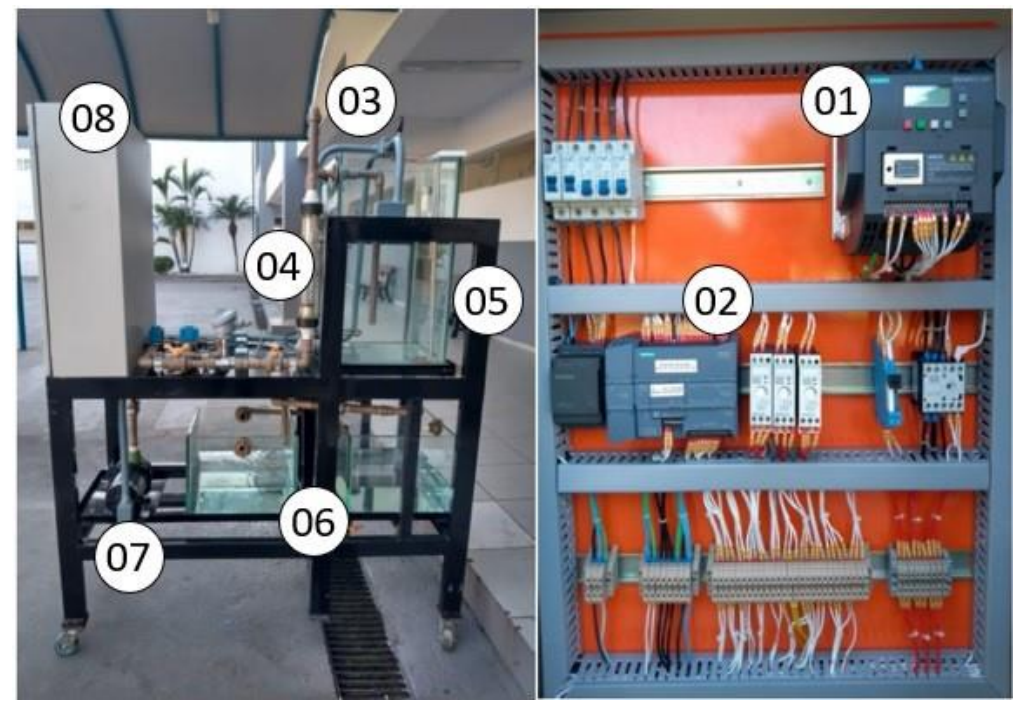

Fonte: SENAI (adaptado).

\subsection{Modelo matemático}

O ponto inicial para a modelagem de um sistema de nível é entender o balanço de massa no tanque, essa variação é dada pela diferença entre vazão de entrada e saída no recipiente, portanto, pode-se dizer que quando a vazão de entrada é igual a vazão de saída, - sistema se encontra em equilíbrio para aquele determinado ponto de operação (AGUIRRE, 2004). Para obtenção do modelo do sistema de nível, tomou-se como base algumas características particulares da bancada. Além disso, o estudo desenvolvido por DUTRA, MILHOMEM e NEVES (2017) auxiliou com a descrição da Equação (1), que demonstra a variação de nível em função das vazões de entrada e saída:

$$
\dot{H}(t)=\frac{1}{A}\left(Q_{e}(t)-Q_{s}(t)\right),
$$

onde $\dot{H}(t)$ é a variação altura de água do tanque em metros, $A$ é a área do tanque em $m^{2}$, $Q_{s}$ é a vazão de saída em $\mathrm{m}^{3} / \mathrm{s}, Q_{e}$ é a vazão de entrada em $\mathrm{m}^{3} / \mathrm{s}$ e $t$ é a variável de tempo em segundos. Na sequência serão definidos os aspectos relevantes do sistema de nível para se determinar as vazões de entrada $\left(Q_{e}(t)\right)$ e de saída $\left(Q_{s}(t)\right)$ do modelo.

A vazão de entrada do sistema de nível depende de dois fatores principais, o primeiro é a bomba utilizada no sistema e a sua capacidade de recalcar água de um reservatório para outro, o segundo fator é a perda de pressão sofrida pelo líquido ao se deslocar pelas tubulações e seus componentes. Sendo assim, a vazão de entrada do sistema é dado por:

$$
Q_{e}(t)=Q_{g} \Delta h(t),
$$

onde $Q_{g}$ é a vazão gerada na saída da bomba em $\mathrm{m}^{3} / \mathrm{s}$, e $\Delta h$ é a perda de carga percentual ao longo da tubulação. Neste caso, é importante observar a característica de funcionamento do atuador, que pode ser vista a partir da Curva Característica da Bomba (ELETRIC e SIEMENS, s.d.), fornecida pelo fabricante do equipamento, que demostra a altura manométrica gerada por determinada vazão e é importante quando se deseja dimensionar um sistema de bombeamento. A curva referente a bomba da bancada pode ser consultada 
em ELETRIC e SIEMENS (s.d.), observando que à medida que a vazão recalcada $(Q)$ aumenta, a pressão de recalque $(P)$ diminui (AGUIRRE, 2013).

Aplicações industriais usualmente utilizam-se de um inversor para variar a velocidade do motor da bomba, que, em geral, é um motor de indução trifásico. Devido a isso, entender a relação entre o inversor e o conjunto motor-bomba é importante para determinar a vazão $Q_{g}(t)$. Assim, buscando-se obter a curva que relaciona a frequência inserida no inversor com a vazão gerada na bomba, realizou-se o seguinte ensaio na bancada de nível, apresentada na Figura 2: 1) Aplicou-se alguns valores de frequência no inversor da bomba e obteve-se as suas respectivas vazões. O ensaio foi repetido por cinco vezes, utilizando-se as mesmas frequências; 2) Fez-se a média aritmética dos dados de vazão obtidos; 3) Utilizando a técnica de interpolação polinomial obteve-se uma curva estimada. As curvas real e estimada podem ser observadas na Figura 3.

Para aproximar a curva simulada da real utilizou-se uma função polinomial de terceiro grau. O software matemático utilizado fornece alguns dados de adequação de ajustes das curvas obtidos através de métodos estatísticos, para demonstrar quão próxima a curva aproximada está da curva real e determinar a qualidade do ajuste (INC, 1994-2021).

Figura 3 - Curva BC-91S - Velocidade vs Vazão.

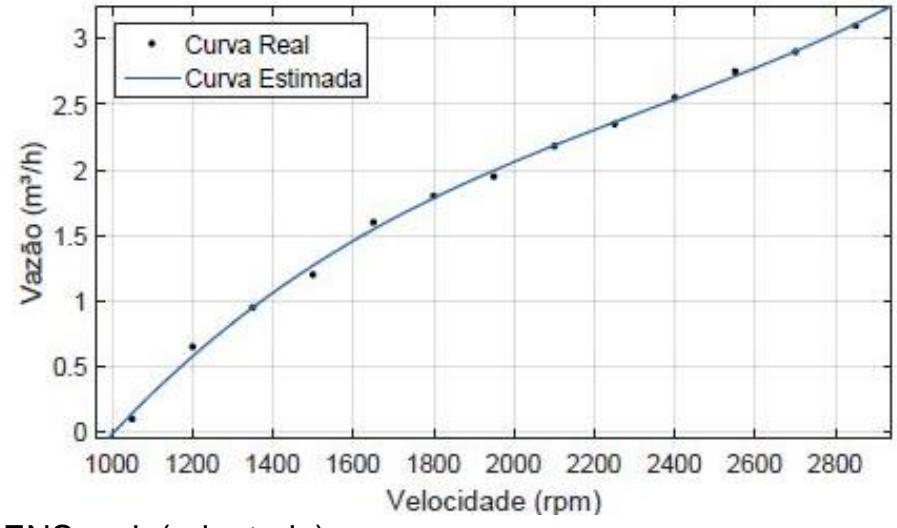

Fonte: ELETRIC e SIEMENS, s.d. (adaptado).

Para essa avaliação são usados: ) a Soma dos Quadrados Devido ao Erro (SSE), quanto mais próximo de zero menor será o componente do erro; ii) o Padrão de Ajuste e o Erro Padrão da Regressão (RMSE), quanto mais próximo de 0 indica um ajuste melhor para a previsão da curva; e o iii) $R$-Square, quanto mais próximo a 1 melhor é a resposta obtida.

Para a curva da Figura 3, os valores obtidos para esses parâmetros são destacados na Tabela 2 - 2.

Tabela 2 - Valores obtidos para os métodos de aproximação entre as curvas.

\begin{tabular}{c|c}
\hline Método Estatístico & Valor Obtido \\
\hline SSE & 0.01918 \\
\hline R-Square & 0.9981 \\
\hline RMSE & 0.04616 \\
\hline
\end{tabular}

Fonte: Autoria Própria.

Através da visualização do gráfico e dos valores obtidos pelos métodos estatísticos, nota-se que a curva encontrada corresponde a uma boa aproximação da curva real. $A$ equação que gera esta curva é descrita por (3)

$$
Q_{g}(x)=p_{1} x^{3}+p_{2} x^{2}+p_{3} x+p_{4}
$$


onde $Q_{g}(x)$ é a função que relaciona a vazão gerada com a frequência $x$ inserida no inversor e $p_{1}, p_{2}, p_{3}$ e $p_{4}$ são $3.997 \times 10^{-10},-2.792 \times 10^{-6}, 0.007657$ e -5.283 respectivamente.

Um dos fatores importantes quanto ao dimensionamento de sistemas é a perda de carga $(\Delta h)$. Quando um fluido se desloca dentro de uma tubulação, ocorre atrito entre o fluido e as paredes do tubo, esse atrito faz com que a pressão existente no tubo diminua na medida que o fluido se desloca, o que influencia diretamente na altura manométrica final da bomba $(H)$ e na sua vazão volumétrica $(Q)$ (GERNER, 2013). Alguns fatores que influenciam na perda de carga são: peso específico do material, diâmetro da tubulação, material dos tubos e conexões, comprimento dos tubos e quantidade de conexões e acessórios. Outro fator que deve ser considerado é a altura geométrica do sistema $\left(h_{g}\right)$, essa é a diferença entre os níveis de sucção e descarga do fluido. O conjunto motobomba deve ser capaz de vencer a altura geométrica e compensar a perda de carga ao longo da tubulação (AGUIRRE, 2013). A pressão que deve aparecer no recalque da bomba para que haja fluxo no sistema é definida na Equação (4). A Figura 4 exemplifica esse conceito

$$
H=h_{g}+\Delta h
$$

Observando (4), para cálculo de perda de carga $\Delta h$, será adotada a Equação (5) de Darcy Weissbach (AGUIRRE, 2013), sendo:

$$
\Delta h=f \frac{L}{D} \frac{V^{2}}{2 g}
$$

em que $L$ é o comprimento equivalente da tubulação $(m), D$ é o diâmetro interno da tubulação $(m), V$ é a velocidade do líquido $(\mathrm{m} / \mathrm{s})$ calculada na Equação $(6), g$ é a aceleração da gravidade e $f$ é o fator de fricção no tubo, a perda de carga na Equação (5) é dada em metros. Para (5), a velocidade do fluido é calculada a partir de (6)

$$
V(t)=\frac{Q(t)}{a},
$$

onde $V(t)$ é a velocidade do fluido, $Q(t)$ é a vazão em $\mathrm{m}^{3} / \mathrm{s}$ e a é a área de seção transversal do tubo em metros (GERNER, 2013).

Figura 4 - Sistema de bombeamento.

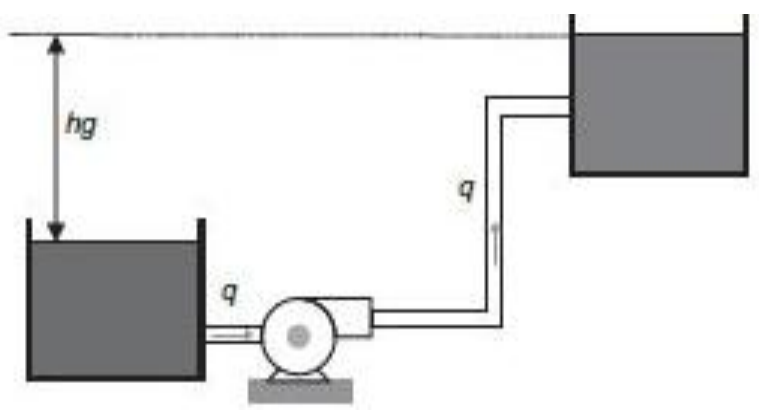

Fonte: Aguirre, 2013 (adaptado).

As tubulações possuem um valor de perda de carga relativa ao seu comprimento em seus trechos retos. Curvas, válvulas e demais componentes da tubulação possuem uma equivalência sobre a perda de carga que causariam se fossem um tubo reto, esses valores dependem do tipo de componente, do diâmetro e do material deste. Existem tabelas já consolidadas que apresentam esses valores. A Tabela 3 demostra os valores equivalentes 
totais das perdas de carga para o sistema utilizado no experimento (Figura 2), considerando o diâmetro interno do tubo de $0,0216 \mathrm{~m}$, e o material tanto dos componentes quanto dos trechos retos como PVC.

Tabela 3 - Perda de carga equivalente.

\begin{tabular}{c|c|c|c}
\hline Componente & Quantidade & Comprimento $(\boldsymbol{m})$ & $\boldsymbol{L}(\boldsymbol{m})$ \\
\hline Curta $90^{\circ}$ & 13 & 0.5 & 6.5 \\
\hline Registro de esfera & 03 & 0.2 & 0.6 \\
\hline Tubulação reta & - & - & 4.8 \\
\hline \multicolumn{2}{c|}{ Comprimento equivalente total } \\
\hline
\end{tabular}

Fonte: Autoria Própria.

Como a vazão do sistema é variável de acordo com a frequência aplicada no inversor, e como a perda de carga $(\Delta h)$ também varia de acordo com a velocidade do fluido, que depende da vazão recalcada (AGUIRRE, 2013), o cálculo do $\Delta h$ deverá ser feito em tempo de execução no simulador. Para isso, considera-se que a altura geométrica $\left(h_{g}\right)$ possui um valor constante na bancada da Figura 2 de $1,2 \mathrm{~m}$, e a vazão de entrada do sistema $\left(Q_{e}(t)\right)$ é o próprio $Q_{g}(t)$ multiplicado ao fator de perda de carga $(\Delta h)$. Assim, a equação que descreve a vazão de entrada para a bancada é definida por (7)

$$
Q_{e}(t)=\left(3.997 \times 10^{-10} x(t)^{3}-2.792 \times 10^{-6} x(t)^{2}+0.007657 x(t)-5.283\right) \Delta h .
$$

Voltando a observar a Equação (1), busca-se agora uma forma para representar a Vazão de Saída $Q_{s}$. Contudo, análises envolvendo sistemas de nível podem ser divididas em duas características considerando o fluxo de escoamento da água, de acordo com o número de Reynolds, podendo ser turbulento ou laminar. Sistemas industriais geralmente possuem o fluxo de líquidos ao longo de tubos e reservatórios, esses processos são em sua maioria turbulentos. Para esse regime, a taxa de fluxo em estado permanente é expressa pela Equação (8) (OGATA, 2011).

$$
Q_{s}=K \sqrt{H(t)},
$$

onde $K$ representa uma resistência empregada pela válvula quanto a passagem do fluido e $H(t)$ é a altura do nível no tanque. Para a válvula da bancada da Figura 2, o valor de $K$ foi obtido através do ensaio de esvaziamento do tanque, que é detalhado a seguir: 1) Encheuse 0 tanque de água até $0.2 m$ de altura; 2 ) Em seguida, a válvula que regula a saída da água foi totalmente aberta e o tanque foi esvaziado; 3) Obteve-se um vetor de nível através do sensor disponível na bancada; 4) Gerou-se a curva que relaciona o nível do tanque partindo da altura máxima até o seu esvaziamento. O gráfico que demostra a curva real e a curva aproximada é apresentado na Figura 5.

Para fins de obtenção do valor $K$ adotou-se fluxo laminar. Realizou-se um ensaio de resposta a entrada nula, onde não há vazão de entrada no tanque. Neste caso, a variação da altura $H(t)$ pode ser modelada como uma exponencial decrescente, onde o valor de $K$ compõe o índice de decrescimento dessa função (OGATA, 2011), assim:

$$
H(t)=C_{1} e^{-\frac{K}{A}} u(t)
$$

onde $C_{1}$ é uma constante relativa a curva do sistema. Comparando a equação obtida com a curva do ensaio na Equação (10) com a Equação (9), nota-se que o valor de $b$ corresponde ao valor de $K / A$ : 
Figura 5 - Ensaio para obtenção do valor K.

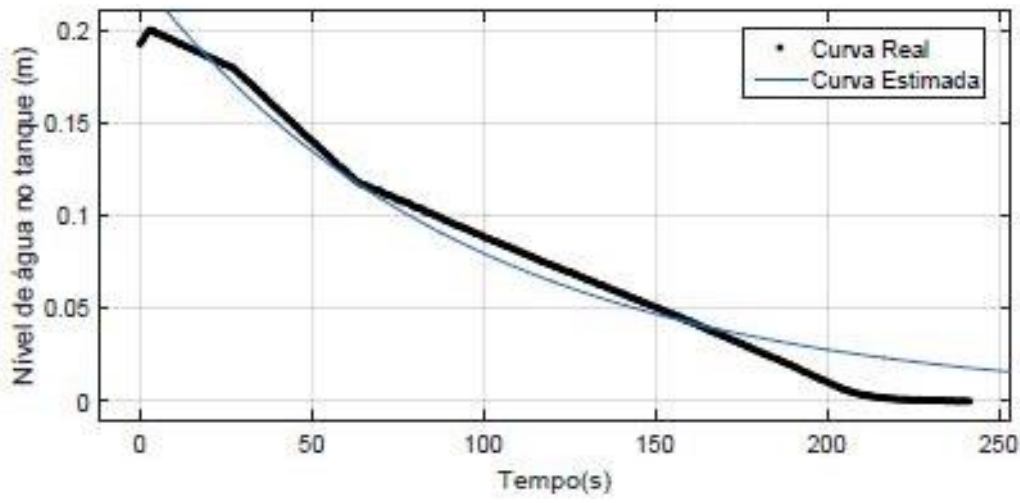

Fonte: Autoria Própria.

$$
y(t)=C_{1} e^{b t}
$$

Dessa forma, assume-se um valor de $b=8.9300 \times 10^{-4}$. O modelo final obtido é demostrado em (11). Ele representa a variação da altura do nível da água no Tanque 1 da Figura 1. Quando uma frequência $(x)$ é inserida no simulador, os valores de vazão de entrada e de saída são calculados e geram uma resposta dinâmica da altura do nível da água no tanque apresentada na Equação (11). Para simplificação da apresentação das equações, a variável $t$ (tempo) será omitida.

$$
\begin{gathered}
\dot{H}=\frac{1}{0.16}\left(\left(\left(3.997 \times 10^{-10} x(t)^{3}-2.792 \times 10^{-6} x(t)^{2}+0.007657 x(t)\right.\right.\right. \\
\left.-5.283) \Delta h)-\left(8.930 \times 10^{-4} \sqrt{H}\right)\right)
\end{gathered}
$$

\section{VALIDAÇÃO DO MODELO}

O simulador está dimensionado para a bancada da Figura 2, portanto, todas as validações e ensaios serão desenvolvidos utilizando-se do modelo específico da bancada. Entretanto, os parâmetros são editáveis no software para que o usuário consiga simular outros sistemas. Para validar o modelo obtido em (11), foi necessário realizar os ensaios de entrada e saída do modelo separadamente. Para validação da vazão de entrada do sistema, o ensaio detalhado a seguir foi realizado: 1) Definiu-se uma altura máxima para o tanque cheio de $0.2 \mathrm{~m}$; 2) Foi inserido um valor de velocidade no inversor de $1800 \mathrm{rpm}$; 3) Utilizando o sensor disponível na bancada obteve-se o vetor relativo ao nível do tanque; 4) Gerou-se um gráfico dos valores obtidos e comparou-se com os dados gerados pelo modelo. O comparativo pode ser analisado na Figura 6.

Figura 6 - Comparativo modelo real vs simulado - À esquerda, vazão de entrada, e à direita, vazão de saída.
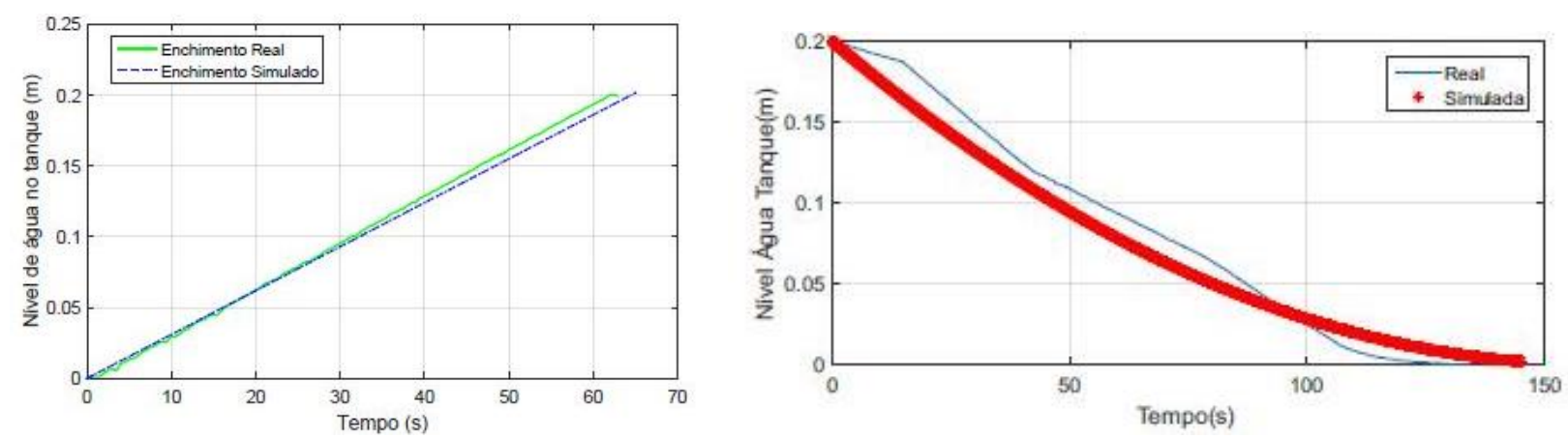

Fonte: Autoria Própria. 
Pode-se analisar através da Figura 6, que as curvas real e simulada são bastante próximas, o que demonstra a satisfatoriedade do modelo encontrado. O tempo de enchimento até $0.2 \mathrm{~m}$ na planta real é de $62 \mathrm{~s}$ enquanto que na planta simulada é de $64 \mathrm{~s}$, como o sistema de nível geralmente é lento uma pequena variação no tempo de enchimento não afeta de forma significativa o sistema.

Para validação do modelo de saída os mesmos passos foram seguidos, porém a vazão de entrada foi considerada nula. O gráfico que demostra esse ensaio está à direita do gráfico de vazão de entrada, na Figura 6. Pode-se observar que a tendência de decrescimento de ambos os sistemas é a mesma, e o tempo de esvaziamento do modelo real é de $135 \mathrm{~s}$ enquanto que no simulador é de $140 \mathrm{~s}$, existindo uma diferença de aproximadamente $5 s$ entre eles, a qual pode ser associada as considerações para cálculo do índice $K$ da válvula.

O hardware utilizado para desenvolvimento do simulador é o CLP S71200 da Siemens, juntamente com as suas ferramentas de programação. O simulador conta também com uma Interface Homem Máquina (IHM) para acompanhamento e utilização das suas funcionalidades. O fluxograma da estrutura do software é apresentado na Figura 7.

Figura 7 - Fluxograma de estrutura do software.

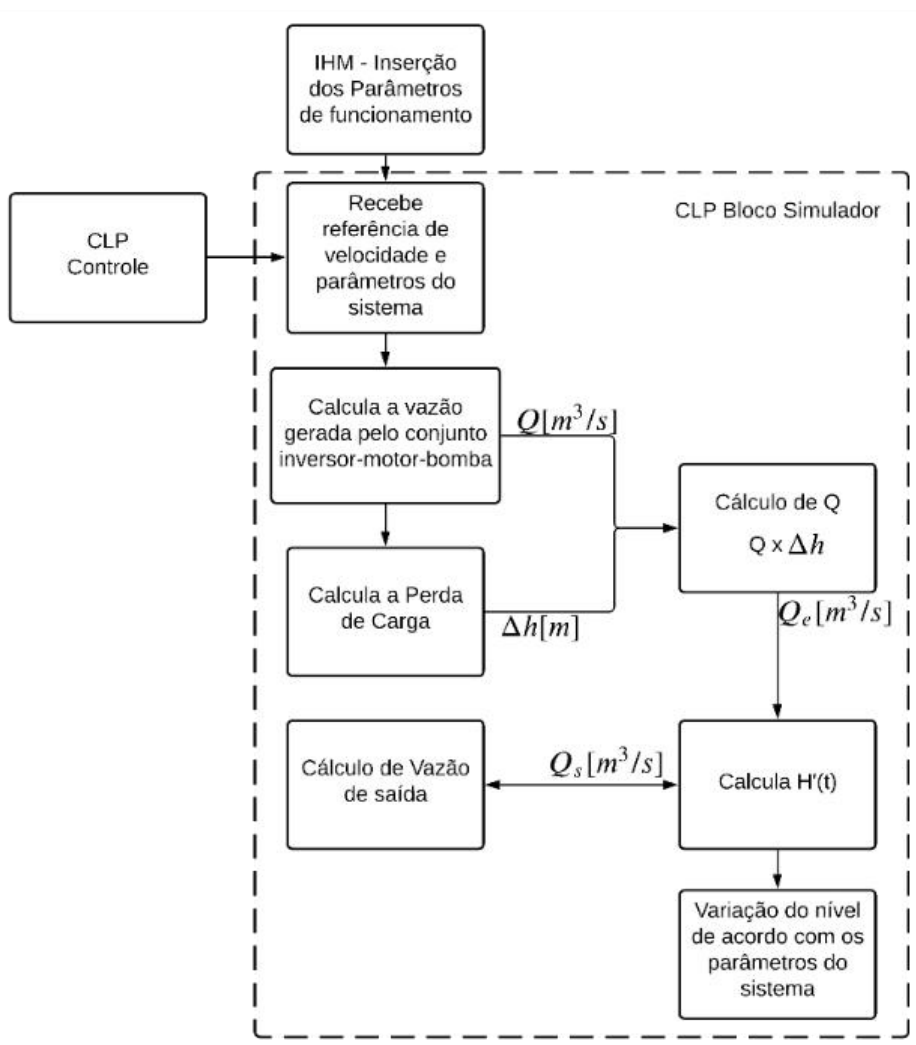

Fonte: Autoria Própria.

O bloco do simulador foi desenvolvido utilizando a linguagem de programação estruturada $S C L$, em um bloco de função (FB). A cada $500 \mathrm{~ms}$ o CLP executa esse bloco, sendo que no primeiro ciclo $(t=0)$ o nível do tanque inicial $(H(0))$ é igual a zero, ou seja, o tanque está vazio, e a cada ciclo o valor de $H$ é incrementado. O software conta com uma (IHM) para dimensionamento do sistema a ser simulado. Os parâmetros que poderão ser editados são definidos na Erro! Fonte de referência não encontrada.4. Os parâmetros $p_{1}, p_{2}, p_{3}, p_{4}$ e $K$ só serão alterados em caso de mudança do sistema utilizado. 
Tabela 4 - Parâmetros editáveis do simulador.

\begin{tabular}{c|c}
\hline Parâmetro & Unidade de Medida \\
\hline Diâmetro interno do tubo & Metros \\
\hline Comprimento total da tubulação (trecho reto) & Metros \\
\hline Tipo de acessórios (Válvula, conexões, etc) & - \\
\hline Quantidade de cada acessório & - \\
\hline Comprimento equivalente acessórios (tabelado) & Metros \\
\hline Área da base do tanque & Metros \\
\hline Equivalência de comprimento em acessórios (G) & Metros \\
\hline
\end{tabular}

Fonte: Autoria Própria

\section{RESULTADOS}

Serão apresentados dois ensaios para verificação dos resultados. Para demonstrar a funcionalidade do software, os parâmetros relativos ao sistema, tais como, área do tanque e velocidade da bomba, serão modificados no software matemático e comparados com as mesmas condições inseridas no simulador. Primeiramente considerou-se a vazão de saída como nula, e modificou-se apenas a frequência inserida na bomba. Enquanto no ensaio de validação da curva a velocidade aplicada era de 1800 rpm (Figura 6, à esquerda), para este ensaio aplicou-se uma velocidade de $2400 \mathrm{rpm}$. O mesmo foi feito para o software matemático. Os demais parâmetros relativos ao tanque não foram alterados.

A altura de enchimento máxima foi mantida como $0.2 \mathrm{~m}$ para que se pudesse verificar que com uma frequência maior na entrada, o tempo até que o tanque atingisse a mesma altura seria menor, e para essa comparação foi possível utilizar o sistema da Figura 2. A Figura 8, à esquerda, demonstra o comparativo das curvas geradas no software matemático, no simulador e na planta real. O tempo de enchimento até a altura máxima foi respectivamente $45 \mathrm{~s}$, $45 \mathrm{~s}$ e $46 \mathrm{~s}$

Para o experimento de esvaziamento, a vazão de entrada é nula. Para verificar a possibilidade de mudança dos parâmetros do tanque, neste comparativo a área do tanque foi modificada para $1 \mathrm{~m}^{2}$ e a velocidade inserida na entrada da bomba foi de $2400 \mathrm{rpm}$. $\mathrm{O}$ tanque foi enchido até uma altura de $0.25 \mathrm{~m}$. Constata-se que para um tanque com área maior, os tempos de enchimento e esvaziamento também deverão ser superiores. Neste caso, utilizou-se a curva gerada para os mesmos valores no software matemático como comparativo. A Figura 8, à direita, demonstra a equivalência entre as curvas.

Figura 8 - Curvas simuladas no CLP e no software - enchimento e esvaziamento.
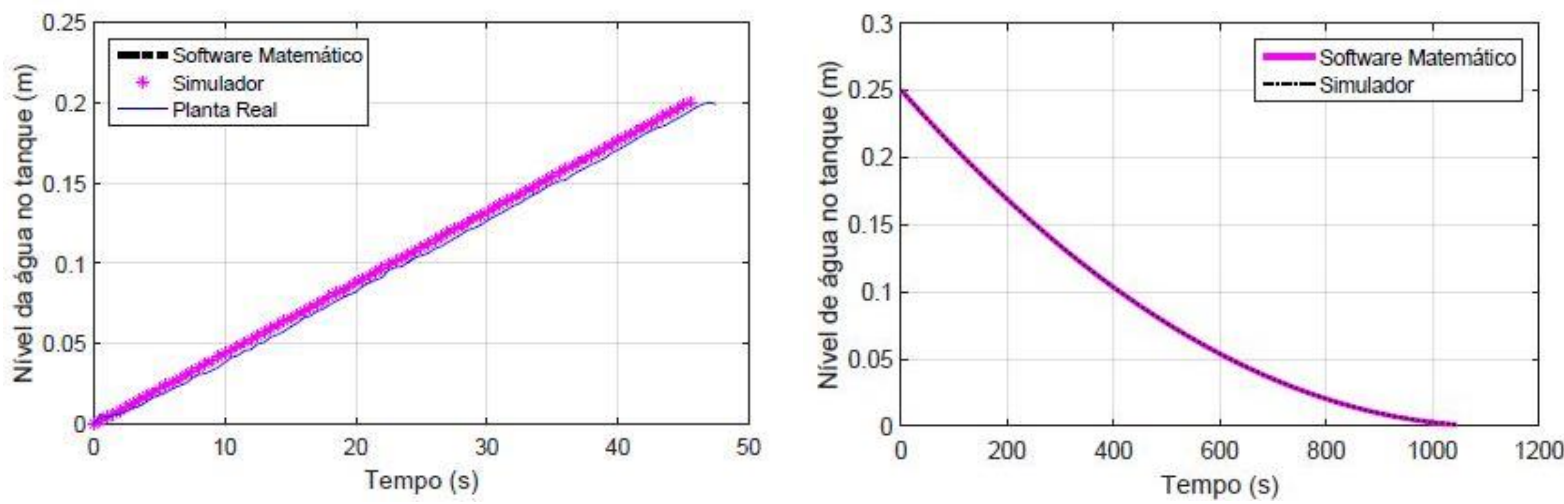

Fonte: Autoria Própria. 
Para facilitar a visualização dessa dinâmica do sistema, o simulador conta com uma interface gráfica, como é mostrado na Figura 9. Os parâmetros atuais do modelo são mostrados na tela com o fundo cinza, os parâmetros que podem ser definidos pelo usuário possuem fundo branco, e o gráfico de barra localizado no tanque aumenta ou diminui de acordo com o nível do recipiente. Ao clicar no botão "gráfico", o gráfico que demonstra a variação da altura do nível $(H)$ ao longo do tempo é exibido, como indica a Figura 9, à direita.

Figura 9 - Interface Homem Máquina - Tela gráfica e Gráficos gerados.
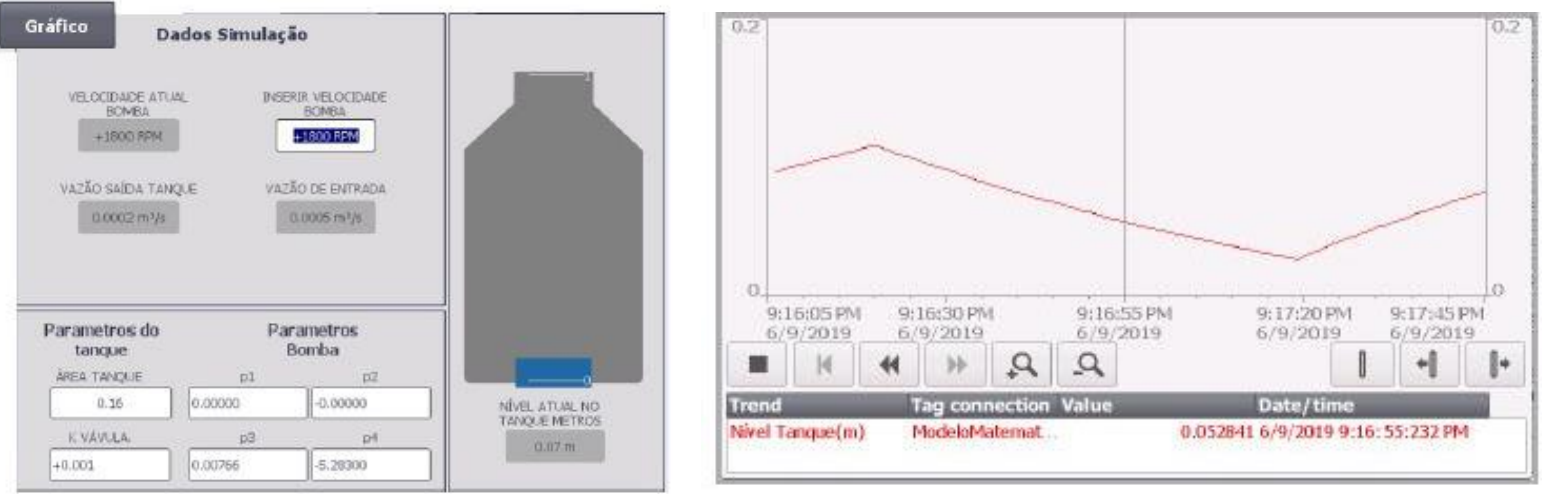

Fonte: Autoria Própria.

A fim de verificar a possibilidade de testar algoritmos de controle na planta simulada, os parâmetros relativos ao tanque foram novamente definidos para os valores da bancada da Figura 2. Dois tipos de controle foram utilizados, um controle Proporcional Integral e Derivativo (PID) e um controle on-off. Para o controle PID utilizou-se o próprio auto ajuste disponibilizado no bloco do CLP para obtenção dos ganhos Proporcional $\left(K_{p}\right)$, Integral $\left(K_{i}\right)$ e Derivativo $\left(K_{d}\right)$. O algoritmo utilizado pelo CLP opera de acordo com (12)

$$
y=K_{p}\left(b \omega-x_{p}\right)+\frac{1}{T_{i} s}\left(\omega-x_{p}\right)+\frac{T_{d} s}{a_{f} T_{d}(s+1)}\left(c \omega-x_{p}\right),
$$

em que $y(t)$ é o valor de saída do algoritmos PID, $K_{p}$ é o ganho proporcional, $s$ é o operador Laplace, $b$ é a ponderação da ação proporcional, $\omega$ é o valor de setpoint, $x_{p}$ é o valor de processo, $T_{i}$ é o tempo de ação integral, $T_{d}$ é o tempo de ação derivativa, $a_{f}$ é o coeficiente de atraso derivativo e $c$ é a ponderação da ação derivativa (SIEMENS, 2003).

Como a variável a ser controlada é a altura da água no tanque, é necessário que o usuário insira o valor desejado de altura $(m)$. Esse dado é chamado de setpoint e representa um valor de referência para o sistema, ou seja, a variação da altura irá ocorrer em torno desse valor. A saída do PID é a velocidade da bomba em rpm, este valor é enviado para a entrada do simulador, que por sua vez, gera uma resposta dinâmica do nível de água no tanque e devolve ao controlador. O gráfico da Figura 10, à esquerda, demonstra 0 funcionamento do PID na planta, onde o setpoint definido é de $0.25 \mathrm{~m}$.

Através do controle on-off, a bomba liga e desliga a medida que atinge o valor de corte do sistema. Esse valor é definido por dois parâmetros, sendo eles o setpoint e a histerese. A histerese é o quanto de variação que poderá ocorrer no sistema partindo do setpoint. $O$ valor de corte é definido então pelo setpoint \pm a histerese. $O$ gráfico da Figura 10 , à direita, demostra o funcionamento desse controle na planta simulada, onde o valor de 
setpoint definido foi de $0.2 \mathrm{~m}$, e a histerese definida foi de $0.1 \mathrm{~m}$. Ao atingir os valores de corte a bomba liga e desliga para manter essa variação.

Figura 10 - Controle PID e Controle on-off.
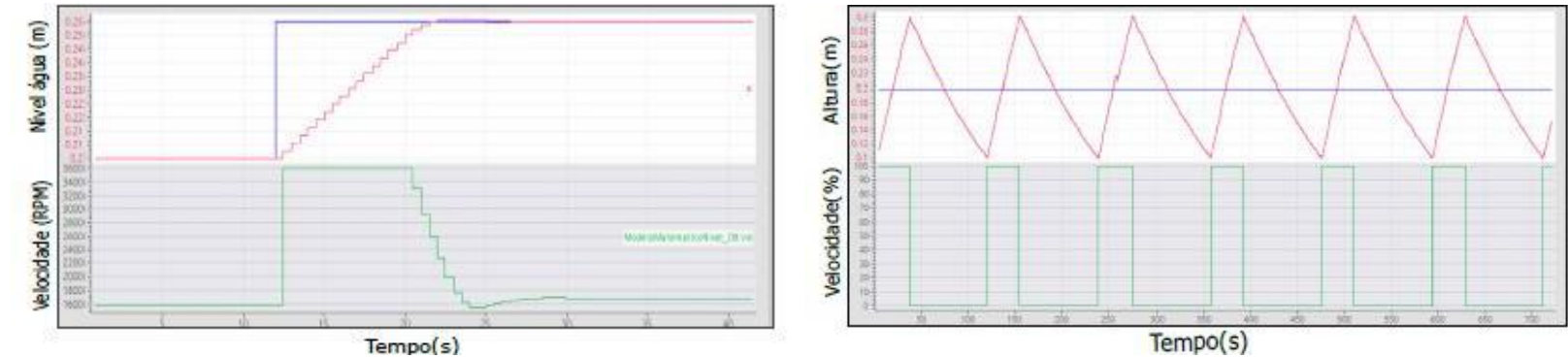

Fonte: Autoria Própria.

Em caso de mudança de sistema a ser simulado, alguns algoritmos deverão ser adotados para obtenção da curva da bomba e do $K$ de resistência da válvula. Depois de obtidos, esses dados poderão ser alterados no software do CLP através da IHM (Figura 9). Para obter o modelo da bomba, basta seguir os passos demonstrados na modelagem matemática para a Vazão de Entrada $Q_{e}$. Do mesmo modo, o processo para encontrar o parâmetro resistência $K$ da válvula é descrito no tópico sobre a Vazão de Saída $Q_{S}$.

\section{CONCLUSÕES}

De maneira geral os objetivos propostos foram alcançados, o modelo encontrado para o sistema de nível descreve de maneira satisfatória o comportamento dinâmico do sistema, pois os resultados obtidos com a bancada demonstraram isso. Um dos principais objetivos do desenvolvimento desse simulador seria a possibilidade de alterar parâmetros e simular outros sistemas do tipo, o emulador possibilita essa troca, porém ainda seriam necessários testes em diferentes tipos de sistemas de nível industriais.

Ao longo do desenvolvimento desse estudo algumas dificuldades foram encontradas, uma delas foi acarretada pela falta de sensores de pressão na bancada utilizada para validar parte dos resultados, o que dificultou a compreensão da influência da Perda de Carga na vazão de entrada do sistema. Outra dificuldade foi adequar as equações discretas a estrutura do CLP, devido a sua lógica de funcionamento.

$O$ estudo desenvolvido possibilita testar algoritmos de controle em bancada, sem a necessidade de se estar presente ao sistema real, a grande vantagem deste em relação aos estudos já desenvolvidos, é a possibilidade de simular diversos sistemas de nível no mesmo software, apenas modificando os parâmetros necessários. Para uma empresa que desenvolve automação de processos variados com nível, essa funcionalidade é desejável.

Para estudos futuros, outros modelos de bombas poderiam ser implementados, como bomba helicoidal e submersa, possibilitando uma gama maior de simulação. Outro fator a ser considerado seria detalhar analiticamente a influência da potência da bomba no sistema simulado, bem como a possibilidade de mudança no líquido utilizado, já que a viscosidade altera as vazões do sistema.

Posteriormente podem ser inseridos ao software do simulador um algoritmo de identificação, como por exemplo MQR, para obtenção dos parâmetros da curva da bomba e do $K$ de resistência da válvula de forma automática.

REFERÊNCIAS

AGUIRRE, Luis Antonio. Fundamentos de Instrumentação. São Paulo: Pearson Education do Brasil, 2013. 
AGUIRRE, Luis Antonio. Introdução à Identificação de Sistemas. Técnicas Lineares e Não-lineares Aplicadas a Sistemas. Teoria e Aplicação. 4. ed, Editora UFMG, 2004.

CARDOSO, Janette; VALETTE, Robert. Redes de Petri. Universidade Federal de Santa Catarina, 1997.

DUTRA, Manoel Kolling; MILHOMEM, Rômulo Lira; NEVES, Carlos Fernando Oliveira Cabeça. Aspectos Práticos Sobre a Modelagem Matemática de Um sistema Didático de Controle de Nível. In: XLV Congresso Brasileiro de Educação em Engenharia, 2017, Joinville. Anais. Joinville. Disponível em: http://www.abenge.org.br/cobenge/sis artigos.php. Acesso em: 04 mai. 2021.

ELETRIC, Franklin; SCHNEIDER. Tabela de seleção de motobombas. Disponível em: https://bityli.com/mR3US . Acesso em: 04 mai. 2021.

GERNER, Valter Rubens. Perda de Carga e Comprimento Equivalente. Senai/SC, 2013.

INC, MatWorks. Matlab Documentation. 1994-2021.

OGATA, Katsuhiko. Entenharia de Controle Moderno. Vol. 5. Pearson, 2011.

SANTORO, Miguel Cezar; MORAES, Luiz Henrique. Simulação de uma linha de montagem de motores. Gestão \& Produção. V. 7, n.3 p. 338-351, 2000.

SIEMENS, Standard PID Control. 3. ed, 2003.

SIEMENS. Inversor SINAMICS V20 - Instruções de funcionamento. Disponível em: https://bityli.com/ToqLO . Acesso em: 04 mai. 2021.

TRIGUEIRO, Tancredo Caciano; LIRA, Valnyr Vasconcelos. SimTank - um simulador de nível de líquido em tanques. Principia. N. 24, p. 112-120, 2014.

\section{INDUSTRIAL PROCESS SIMULATOR FOR CONTROL ALGORITHM TESTING}

Abstract: Much of the time spent in the development of automation systems is in the implementation period, because for this system to be operational it is necessary to perform several tests. A very useful tool that could reduce this time is the simulator, used in several areas, it allows the testing and validation of a process without the need to be close to the real system. Under this context, the article presents a simulator developed in a Programmable Logic Controller capable of allowing the user to predict the behavior of a process facing the control algorithm inserted, which would allow a reduction in the control implementation time in industries. The simulator receives input signals from a second Programmable Logic Controller and sends a dynamic response to it. The main feature of this simulator is to allow the user to change specific parameters according to the system to be simulated. For this, it has an interface where the user can not only change these parameters, but also observe the generated behavior.

Keywords: Industrial Applications, Process Automation and Control, Process Simulators, Programmable Logic Controller 